\title{
SFED recommendations for IBD endoscopy during COVID-19 pandemic: Italian and French experience
}

\author{
Federica Furfaro, Lucine Vuitton, Gionata Fiorino, Stephane Koch, \\ Mariangela Allocca, Daniela Gilardi, Alessandra Zilli, Ferdinando D’Amico, \\ Simona Radice, Jean-Baptiste Chevaux, Marion Schaefer, Stanislas Chaussade, \\ Silvio Danese (iD) and Laurent Peyrin-Biroulet (D)
}

\begin{abstract}
The current coronavirus disease 2019 (COVID-19) pandemic caused by severe acute respiratory syndrome coronavirus 2 (SARS-CoV-2) infection has required a complete change in the management of patients with inflammatory bowel disease (IBD) who need to undergo endoscopic procedures. Several preventive measures must be taken to avoid the spread of infection among health-care professionals and patients with IBD, including the use of personal protective equipment, greater attention to endoscopic room hygiene and rescheduling of non-urgent procedures. This Perspective aims to provide a guide based on the Italian and French experience to better face the difficulties encountered by endoscopists during this global health emergency. In particular, recommendations regarding the use of personal protective equipment to prevent COVID-19 transmission, both for patients and health-care professionals, are proposed and different scenarios in endoscopic IBD management are evaluated to suggest when endoscopy could be rescheduled and replaced by alternative biomarkers.
\end{abstract}

Endoscopy is the mainstay for the diagnosis and management of patients with inflammatory bowel disease (IBD). Full assessment of disease activity, severity and extension as well as the assessment of mucosal inflammation, surveillance colonoscopy for colorectal cancer or dysplasia, and endoscopic management of bowel obstruction represent the essential quality standard of care ${ }^{1,2}$. The coronavirus disease 2019 (COVID-19) pandemic, caused by severe acute respiratory syndrome coronavirus 2 (SARS-CoV-2) ${ }^{3}$ infection, a coronavirus first detected in China in late December 2019 (REF. ${ }^{4}$ ), is currently overwhelming health-care systems worldwide and requires a fundamental restructuring of primary care and hospital activities $^{5}$. As of 4 June 2020, according to the World Health Organization (WHO), there have been $6,416,828$ confirmed cases and 382,867 deaths worldwide, with 233,836 confirmed cases in Italy (33,601 deaths) and 151,677 confirmed cases in France $(29,021 \text { deaths })^{6}$. Alongside measures to protect health-care professionals (HCPs) from COVID-19, several hospitals have now adopted different measures to protect patients from being infected while at hospital. These measures include checkpoints to assess symptoms and signs suggestive of COVID-19, a reduction or suspension of elective procedures, and the provision of personal protective equipment (PPE) for patients.

SARS-CoV-2 is a new coronavirus, very similar to SARS-CoV and MERS-CoV (Middle East respiratory syndrome coronavirus) $)^{3,7}$ and with similar ways of infection and complications, although SARS-CoV-2 seems to be more contagious than SARS-CoV ${ }^{8}$. The main route of SARS-CoV-2 infection is aerosolized droplets that are expelled during coughing, sneezing or breathing, but there are also concerns about possible airborne transmission ${ }^{9}$. Furthermore, viral RNA can be detected in faeces, raising concerns about faecal-oral transmission ${ }^{10,11}$. However, evidence on the gastrointestinal involvement of SARS-CoV-2 is limited and with conflicting results. In a detailed virologic analysis of nine cases, Wölfel et al. isolated infectious virus from throat, lung and sputum samples but rarely from stool samples, despite high virus RNA concentrations in faeces ${ }^{12}$. Indeed, extrapulmonary detection of viral RNA does not mean that infectious virus is present. Sputum and stool RNA concentrations declined more slowly than throat swab RNA concentrations and remained positive for more than 3 weeks in six of nine patients despite full resolution of symptoms. Nevertheless, despite the failure to isolate live SARS-CoV-2 from stool, probably due to the mild course of cases included in the study, with only one case with diarrhoea, researchers support the possible replication of the virus in the gastrointestinal tract; this aspect requires further study ${ }^{12}$. In a research letter, Wang et al. analysed different specimens (collected from blood, sputum, stool, urine and nasal swab) from 205 patients with COVID-19 and described the detection of live virus in stool, suggesting that SARS-CoV-2 could be transmitted by the faecal route ${ }^{13}$. Reported in a brief communication, Xiao et al. tested for SARS-CoV-2 RNA in stool in 73 hospitalized patients infected with SARS-CoV-2 (REF. ${ }^{14}$ ). More than $50 \%$ of patients had a positive faecal test, specifically SARS-CoV-2 RNA in stool and, in some cases $(23.29 \%)$, the test continued to be positive despite a negative nasal swab until 12 days after the onset of the first symptom; the investigators did not report on viable virus extraction from faecal samples ${ }^{14}$. A commentary also highlights two unpublished studies that report the presence of SARS-CoV-2 in stool specimens of patients with COVID-19 infection $^{15}$. Interestingly, Ong et al. reported a typical example of the potential for faecal transmission of SARS-CoV-2 (REF. ${ }^{16}$ ). 
The researchers collected samples from the bathroom of a patient with the confirmed presence of SARS-CoV-2 in stool by reverse transcriptase PCR (RT-PCR) and no diarrhoea. Samples from the surface of the toilet bowl and sink were positive and post-cleaning samples were negative, suggesting that stool could be a potential route of transmission ${ }^{16}$.

SARS-CoV-2 likely infects the gastrointestinal epithelium, producing virions secreted in the stool by the infected gastrointestinal cells ${ }^{14}$. Importantly, Lamers et al. demonstrated, using an experimental model of human small intestinal organoids and confocal and transmission electron microscopy, that SARS-CoV-2 infects and replicates within enterocytes in vitro and that the intestinal epithelium supports virus replication ${ }^{17}$. However, whether the viral concentration of SARS-CoV-2 in stool is sufficient for the transmission of infection remains currently unclear. Furthermore, the presence of SARS-CoV2 RNA in stool specimens can be independent of the presence of diarrhoea or other gastrointestinal symptoms (anorexia, nausea, vomiting, abdominal pain $)^{18}$. Nevertheless, there are cases of patients with symptoms and lung imaging compatible with COVID-19 but with negative pharyngeal swab and positive stool real-time RT-PCR for SARS-CoV-2 $\left(\right.$ REF. $\left.^{19}\right)$. Thus, both routes, airborne transmission and faecal-oral transmission, should be considered in understanding and reducing the risk of infection during endoscopic procedures. According to a study in the United States, the rate of infection in endoscopy units normally ranges from 1.1 for screening colonoscopies to 3.0 for oesophagogastroduodenoscopy for every 1,000 procedures $^{20}$. The goal of protective measures in the endoscopy setting should be to maintain the risk below these numbers during the COVID-19 pandemic. Currently, it is not clear whether endoscopic procedures (particularly colonoscopy) are of high risk for COVID-19 transmission. Considering that the available evidence is quite slim, we suggest the use of all disposable protective measures to prevent infection transmission.

In this Perspective, we provide some guidance from the Humanitas Research Hospital (Rozzano, Milan, Italy) and the Société Française d'Endoscopie Digestive (SFED) for performing endoscopy in patients with IBD during the COVID-19 pandemic based on the Italian and French experience. Recommendations regarding the use of PPE to prevent COVID-19 transmission are proposed and different scenarios in endoscopic IBD management are evaluated. The recommendations discussed are based on the available evidence and expertise as there are limited data with respect to COVID-19 in patients with IBD with or without gastrointestinal symptoms. In the past few months, different societies and experts have already proposed advice or recommendations to manage endoscopy and/or patients with IBD during the COVID-19 pandemic, including the American Gastroenterological Association (AGA; recommendations for gastrointestinal procedures) ${ }^{19}$, the British Society of Gastroenterology ${ }^{21}$, the International Organization for the Study of IBD (recommendations for patients with IBD $)^{22}$, the European Society of Gastrointestinal Endoscopy and European Society of Gastroenterology and Endoscopy Nurses and Associates (Position Statement on gastrointestinal endoscopy $)^{23}$, the Chinese Society of IBD (guidance for patients with pre-existing digestive diseases) ${ }^{24}$, the Asian Pacific Society for Digestive Endoscopy ${ }^{25}$, a panel of experts in the United States ${ }^{26}$ and a panel of international experts ${ }^{27}$. Currently, the available evidence on COVID-19 in the context of IBD, as well as in relation to reported gastrointestinal symptoms, is very limited. Accordingly, the recommendations proposed presuppose that everyone (both patient and HCPs) are at high risk - the recommendations will probably evolve as the pandemic progresses.

\section{Protection against COVID-19 infection Protection of patients: admission} The data about the spread of COVID-19 in countries that applied strict containment measure ${ }^{28}$ suggest that the risk of being infected is higher at the community level than in hospitals. Thus, the first protection measure is to avoid having potentially infected individuals admitted to the hospital and endoscopy rooms. Furthermore, the infection of patients with IBD by HCPs must be avoided. Although COVID-19 can be transmitted by the $1-3 \%$ of the affected individuals who are asymptomatic ${ }^{29}$, symptomatic patients remain the main source of infection. Patients with IBD who need endoscopy should be checked for typical symptoms of COVID-19 such as fever, cough, dysgeusia, dysosmia and dyspnoea. The most challenging COVID-19 symptoms might be gastrointestinal symptoms, including abdominal pain, nausea, vomiting and/or diarrhoea, occurring in a reported 3-79\% of individuals ${ }^{18,29}$, which can be present in patients with IBD but are rarely the only symptoms in patients with COVID-19 (REF. ${ }^{30}$ ). The WHO report from China indicates that, within a median time of 5-6 days after infection (range 1-14 days), these patients can develop fever and respiratory symptoms (usually mild) ${ }^{29}$.

At Humanitas Research Hospital and in French centres following guidance from the SFED, endoscopic procedures were reduced, limiting access to the centre to patients with IBD for whom endoscopy is necessary and not deferrable. To postpone elective surgeries and endoscopies at this time is also suggested in the International Organization for the Study of IBD recommendations ${ }^{22}$. In particular, endoscopy is performed for patients with a new clinical flare both in ulcerative colitis and Crohn's disease, when delay is not recommended (that is, to rule out superinfections in severe flares refractory to steroids), or in patients with IBD with dysplastic lesions or polyps for whom endoscopic resection was already planned.

A nasal swab, as with screening procedures prior to endoscopic examinations, could be an option, although fast-track SARS-CoV-2 tests are not generally validated and false-negative tests (up to $30 \%)^{31}$ might provide false reassurance. At Humanitas Research Hospital, patients who have a scheduled endoscopy are contacted by phone the week before and again 1-2 days before the procedure to identify patients at risk of having COVID-19 prior to the commencement of bowel cleansing and before their arrival at the hospital. If a patient refers to specific symptoms, such as fever $\left(>37.5^{\circ} \mathrm{C}\right)$, cough, dysgeusia, dysosmia and dyspnoea, or if a patient has been in contact with a confirmed case of COVID-19 (confirmed by testing) or with individuals highly suggestive of COVID-19 infection, HCPs can decide to postpone the endoscopic examination and reschedule it, according to clinical condition. Furthermore, patients can be advised to perform specific tests, such as a nasal swab, or to contact the green number for a COVID-19 emergency (a specific telephone COVID-19 emergency service in Italy).

For patients accessing the endoscopy service (FIG. 1), a checkpoint is available at every public entrance of the hospital (in Italy) and at the entrance of the endoscopy unit (in France) to assess body temperature and current and/or previous symptoms of COVID-19. In Italy, the endoscopist or the endoscopy nurse must double-check the same information before the patient enters the endoscopy room. At the checkpoints, HCPs are also evaluated, assessing body 
temperature and COVID-19-related symptoms, and dedicated staff provide everyone with a simple surgical mask and an alcoholic solution to clean hands, on the assumption that there could be asymptomatic carriage of the infection. In both Italy and France, patient relatives and caregivers are strictly forbidden from entering the hospital before the endoscopic procedure and are stopped at the checkpoints unless the patient requires specific assistance such as for patients in a wheelchair or relatives of patients under 18 years of age. Medical and nurse students are not allowed in the endoscopy units during the pandemic crises. Additionally, appointments for endoscopy procedures must be organized to avoid crowding in the waiting room and to maintain a reasonable distance between patients; if possible, only one patient will be in the waiting room at a given time.

\section{Protections: endoscopy room}

In the endoscopy room, both the patient with IBD and HCPs must be protected. Potentially, there is a risk of transmission from the patient to the endoscopist and from the endoscopist or other HCPs to the patient. The use of adequate PPE, in particular masks, protects from the risk of being infected by respiratory viruses, with no notable differences between surgical masks and N95 masks (FFP2 in Europe) in a randomized clinical trial in the context of seasonal human coronaviruses (but not specifically SARS-CoV-2), influenza viruses and rhinoviruses ${ }^{9,32}$. In March 2020, the $\mathrm{AGA}^{19}$ and the SFED ${ }^{33}$ recommended the use of N95 (FFP2) masks, instead of surgical masks, to protect HCPs during upper and lower gastrointestinal procedures, regardless of the COVID-19 status of the patient (with low to moderate certainty of evidence). A meta-analysis of retrospective studies demonstrated an increased risk of COVID-19 transmission during aerosolgenerating procedures (upper endoscopy), but the panel of experts extended this recommendation to lower gastrointestinal procedures $^{19}$. The panel of experts considered possible aerosolization during colonoscopy, in particular during the insertion and removal of instruments through the biopsy channel and the presence of the virus in the stool and advised on the use of N95 masks for lower gastrointestinal procedures as a precautionary measure to protect the endoscopist from the risk of possible COVID-19 transmission from the patient if infected by SARS-CoV-2 $\left(\right.$ REF. $\left.^{19}\right)$. Unfortunately, in clinical practice, it could be difficult to have these kinds of PPE for every procedure; in this instance, the AGA suggests the use of the same N95 mask all day long and changing a surgical mask covering the N95 mask ${ }^{19}$. The SFED suggests changing the mask once at midday and practicing endoscopy with the minimal mandatory staff (doctor and nurse) ${ }^{33}$. The usual procedures of disinfection and decontamination by neutral detergent and viricidal disinfectant, $0.05 \%$ sodium hypochlorite or $70 \%$ ethanol of surfaces and devices are effective in clearing the virus ${ }^{34,35}$. The major issue is the possibility that the virus can remain alive and contagious in aerosols, as van Doremalen et al. demonstrated that SARS-CoV-2 remained viable in aerosols for at least $3 \mathrm{~h}$, with a reduction in infectious titre from 103.5 to $102.7 \mathrm{TCID}_{50}$ (median tissue culture infectious dose) per litre of air $^{34}$. To avoid this risk, at Humanitas, patients and HCPs wear masks during the entire procedure and during the entire time spent at the hospital.
In the endoscopy room, entry is restricted to the endoscopist, the nurse and the patient. Considering that the endoscopy staff are at higher risk of infection during the procedures and could spread the virus, regular patient care (such as infusion management) is separated from the endoscopy unit, with other separate, dedicated staff, to decrease risk of viral transmission. During the procedure in the endoscopy room, the patient dresses in a cotton gown, a hairnet and a surgical mask (FIG. 2). HCPs must remove contact lenses, if present, and must dress following this specific order: a hairnet, a long water-resistant gown (in blue in FIG. 2) with back closure, a N95 (or FFP2) mask, goggles for eye protection and over-sleeve gloves over the gown (the surgical gown, not because of sterility, but because they are longer than others and can cover the skin up to the wrist). To avoid becoming infected and/or passing on potential infection to different patients, before a patient's entrance into the endoscopy room, HCPs must wear, over the other layers, a single-use gown (in white in FIG. 2) and a pair of nitrile gloves (in blue in FIG. 2, double gloves are also recommended in the AGA recommendations $\left.{ }^{19}\right)$. HCPs change the white disposable gown and the blue gloves for each procedure, as is normal in clinical practice outside of the COVID-19 era. The SFED recommends long waterresistant gowns only for HCPs caring for patients who are COVID-19-positive and disposable plastic gowns when treating other patients ${ }^{33}$.

Before and after all patient interaction and before putting on and removing the PPE, hand washing (keeping the surgical gloves on, as if they were HCP's own skin) with water and soap or alcohol-based

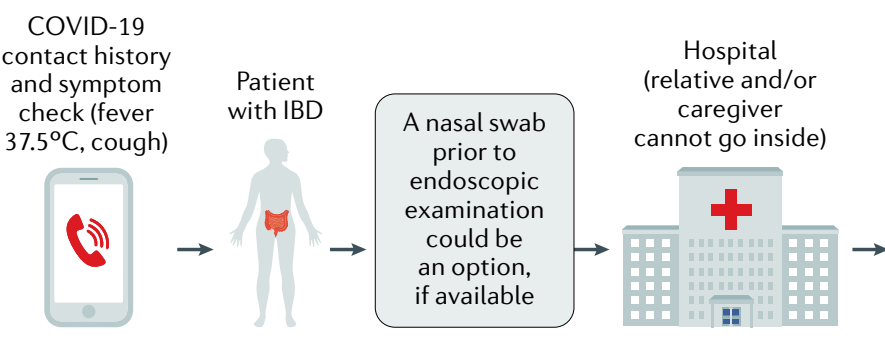

Fig. 1 | Admission of patients with IBD for endoscopy during COVID-19 pandemic. Health-care professionals contact patient with IBD by phone to check for coronavirus disease 2019 (COVID-19) symptoms and/or to check whether a patient has had contact with a test-confirmed case or suspected case of COVID-19 to identify patients at risk of having COVID-19. Depending on the response, the decision could be made to postpone the exam. A nasal swab prior to endoscopic examination could be an option, although fasttrack SARS-CoV-2 tests are not yet validated and false-negative tests (up to $30 \%$ ) can provide false reassurance and therefore a nasal swab is not

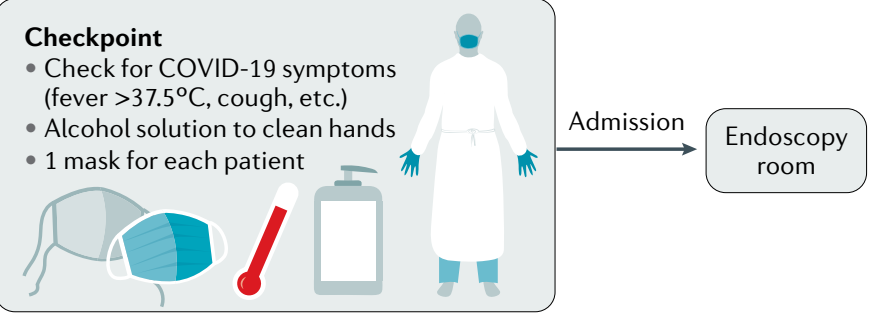

currently recommended by the SFED nor European or American guidelines for endoscopy. Relatives and caregivers of patients are forbidden from entering the hospital. At the hospital entrance and/or at the endoscopy unit a checkpoint is available to assess body temperature and current and/or previous symptoms of COVID-19. A dedicated staff member provides all attendees with a mask and an alcoholic solution to clean hands. Upon completion of these checks, the patient can be admitted to the endoscopic room. IBD, inflammatory bowel disease; SARS-CoV-2, severe acute respiratory syndrome coronavirus 2; SFED, Société Française d'Endoscopie Digestive. 


\section{PERSPECTIVES}
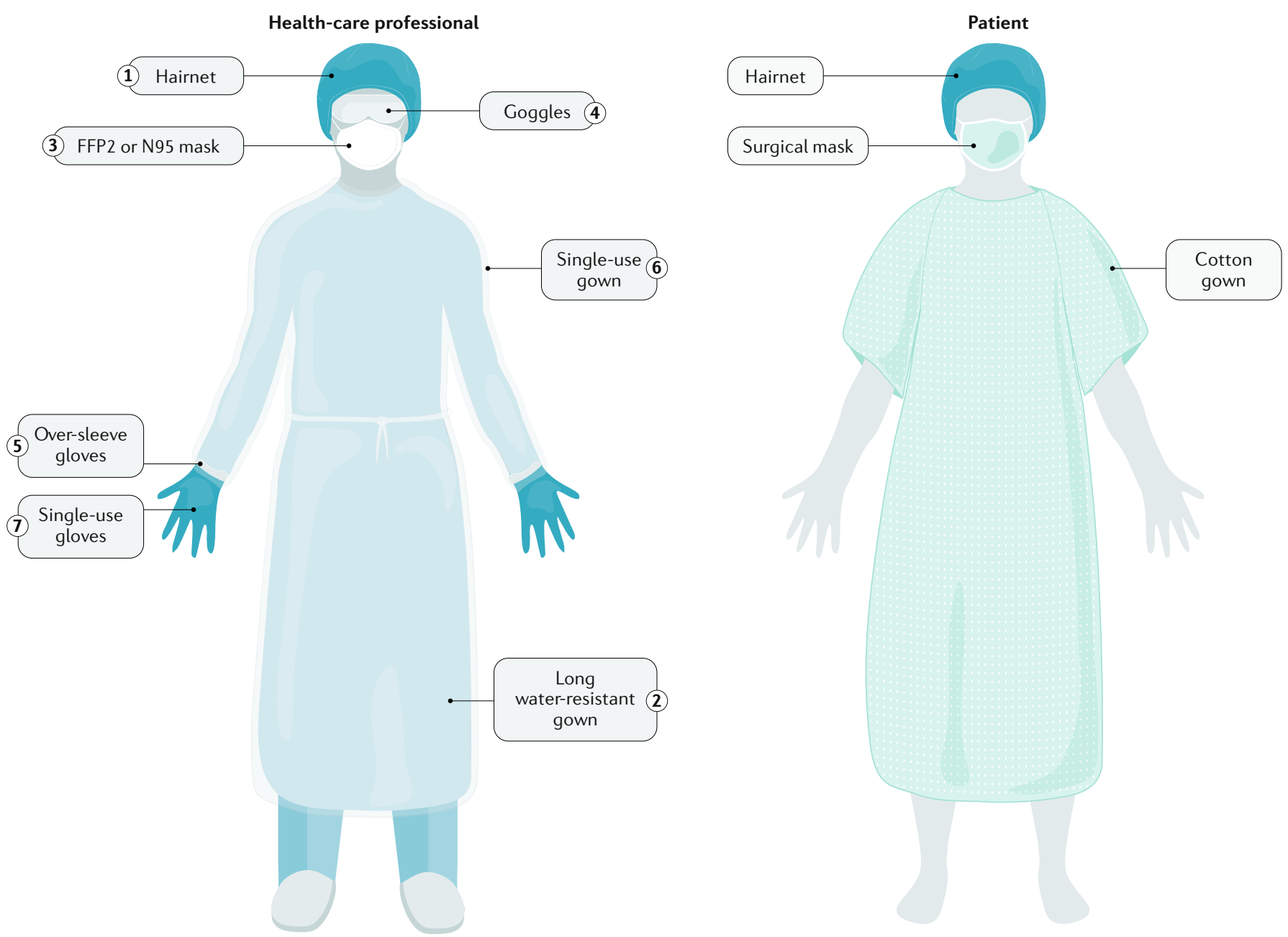

Fig. 2 | PPE for patients with IBD and health-care professionals in the endoscopy room. Personal protective equipment (PPE) is essential during the coronavirus disease 2019 (COVID-19) pandemic to reduce the risk of transmission in hospital settings. In the endoscopy room, the patient dresses in a cotton gown, a hairnet and a surgical mask. Health-care professionals must dress following this specific order (as numbered in the figure): a hairnet, a long water-resistant gown (in blue in figure) with back closure, an N95 or FFP2 mask, goggles for eyes protection, over-sleeve gloves over the gown, a single-use gown (in white in figure) and a single-use pair of nitrile gloves (in blue in figure). IBD; inflammatory bowel disease. hand solution is mandatory. When anaesthesiology is mandatory for sedation, the SFED advises orotracheal intubation to protect the endoscopy team from COVID-19 aerosols ${ }^{33}$. Nevertheless, because of the pandemic, it is very likely that anaesthesiologists would not be available for deep sedation because they are attending to patients with COVID-19 in the intensive care unit. Thus, conscious sedation remains the most feasible option and can be provided and managed even though the patient is wearing a mask.

At Humanitas, in the COVID-19 inpatient department, we have negativepressure rooms (airborne isolation rooms) to prevent generated aerosols from diffusing outside the room. Unfortunately, we do not have a negative-pressure room in the endoscopy department and so emptying the endoscopy room of all non-used equipment is essential to facilitate room cleaning and disinfection. The use of air renewal in the operative room is the most protective tool ${ }^{27}$. The timing to open the endoscopy room for air renewal depends on the adequacy of PPE worn by patients and HCPs. On the other hand, in several centres, scarce availability of and access to PPE have been reported; centres using surgical masks for PPE should open the endoscopy room for more time to allow air renewal. In the IBD endoscopy room, we do not perform room disinfection after each procedure for COVID-19-negative patients, rather dedicated staff perform a standard room disinfection using 1:100 dilution of household bleach and water once a day as a standard decontamination process. After each procedure, all endoscopes and reusable accessories are reprocessed with a standardized and uniform reprocessing procedure. Kampf et al. demonstrated that human coronaviruses can be efficiently inactivated by surface disinfection procedures with specific agents ${ }^{36}$. We could assume that these methods would work for SARS-CoV-2, indicating that current endoscope disinfection techniques could be sufficient to prevent COVID-19 infection $^{36}$. Beds are also cleaned with specific disinfection products and bed sheets are changed for each patient. Finally, avoiding viral dissemination to patients with IBD who are potentially immunocompromised because of therapies (steroids, immunosuppressants or immunomodulators) is particularly important. Given that nurses and physicians are at particular risk of contamination and could also be asymptomatic carriers of SARS-CoV-2, maintaining physical distance wherever possible, hand cleaning and the wearing of surgical masks outside the endoscopy units should always be the rule at any time of the day at the hospital for all HCPs. 
For patients with IBD with known or presumptive COVID-19 with non-deferrable endoscopic procedures we follow the same precautions used for non-IBD patients with COVID-19 (REF. $\left.{ }^{33}\right)$. The procedure should be performed in a dedicated room and the exams must be concentrated in the last part of the day to enable the correct room cleaning and disinfection protocol after the endoscopic procedure. The patient has to be transferred to the endoscopy room at a specific time, without encountering obstacles. Once the patient is in the endoscopy room the door will be closed until the end of the procedure ${ }^{33}$. There is a specific protocol for wearing and removing PPE, with a specific order as noted earlier. The PPE has to be donned in this order: hairnet, gowns, filtering face-piece (N95 or FFP2 mask), goggles for eye protection, 2 pairs of gloves. At the end of the endoscopy procedure, the gloves and gown should be the first to be removed and a new pair of nitrile gloves should be worn to prevent self-contamination. Subsequently, the PPE has to be removed in this order: goggles, respiratory protection, hairnet and, finally, the new pair of gloves worn. At the end of the examination, the removal of the PPE from patients with COVID-19 is crucial to protect HCPs from contamination.

\section{Patient protection: follow-up}

In late February 2020, the WHO reported the development of COVID-19 symptoms after a median time of 5-6 days post-infection of SARS-CoV-2 (range 1-14 days) ${ }^{29}$. In a cohort of 40 admitted hospital patients in Wuhan, Huang et al. reported a median time from illness onset to dyspnoea of 8 days in $55 \%$ of patients ${ }^{37}$. Patients undergoing endoscopy could therefore develop symptoms after the procedure in instances in which they have contracted the SARS-CoV-2 infection at the community level just before endoscopy. Therefore, IBD nurses perform a follow-up call at least 1 week after the procedure to identify potential new COVID-19 cases and to verify whether the protective measures have worked in avoiding contamination and dissemination in the endoscopy environment; of note, the patient can contract the disease elsewhere once outside the hospital setting.

\section{Management of patients with IBD Monitoring disease activity}

Patients with IBD normally undergoing planned endoscopies to monitor endoscopic response to therapy or to evaluate endoscopic disease activity could be managed instead by checking clinical activity with patient-reported outcomes (PROs; for instance, number of bowel movements and abdominal pain for Crohn's disease or bowel movements and rectal bleeding for ulcerative colitis) and non-invasive tests such as stool and blood exams and cross-sectional imaging. Indeed, all protective measures already described can limit, but not exclude, the risk of infection for patients during endoscopy: patients can be at risk of SARS-CoV-2 infection whilst travelling to the hospital (that is, by public transportation or potentially by use of public toilets). Whether alternative non-invasive tools can replace endoscopy, at least for the monitoring of patients under therapy, has been a topic of debate since before the COVID-19 pandemic. Cross-sectional imaging, such as bowel ultrasonography or magnetic resonance enterography (MRE), are valid alternatives; however, they also require hospital attendance, with similar challenges as with endoscopy in protecting patients and HCPs from SARS-CoV-2. The use of blood tests and faecal biomarkers could play a key role in avoiding endoscopy when this procedure is not urgent or necessary (FIG. 3). The CALM trial ${ }^{38}$ demonstrated that treatment escalation and tight control based on levels of faecal calprotectin and serum C-reactive protein (CRP), compared with treatment based on clinical management, was associated with a statistically significant higher proportion of patients with Crohn's disease achieving endoscopic remission at week 48 (46\% versus 30\%, 95\% CI 3.9-28.3; $P=0.010)$. In patients with ulcerative colitis, faecal calprotectin levels had a statistically significant positive correlation with endoscopic extent, mucosal healing and histological activity ${ }^{39,40}$. In this context, the use of home tests for faecal calprotectin levels to avoid manipulation of stools and virus in the laboratory is of particular interest. Home faecal calprotectin tests, where available, together with remote monitoring of symptoms and PROs, could be a safe alternative to monitor patients by avoiding endoscopies.

Patients with IBD with onset of gastrointestinal symptoms during COVID-19 pandemic, such as abdominal pain and diarrhoea, will need to undergo specific examinations to identify the correct diagnosis. First, the diagnosis of COVID-19 will be considered, as SARS-CoV-2 infection can occur with gastrointestinal symptoms. In cases of gastrointestinal symptoms in COVID-19, the median duration of symptoms is 4 days (range 1-9 days) ${ }^{41}$ and thus shorter than an IBD flare, which typically does not resolve without therapy. Evidence of concomitant fever, cough, dysosmia and fatigue can help in the clinical diagnosis and should prompt nasal swab and PCR testing for SARS-CoV-2. The second step is to exclude usual infectious diseases, such as Salmonella, Shigella, Campylobacter, Clostridioides difficile (formerly known as Clostridium difficile) or parasitological infection, by performing microbiological analysis of stool samples. Finally, faecal calprotectin can help discriminate between IBD and irritable bowel syndrome, keeping in mind that it could be elevated in cases of COVID-19 infection, as reported in a letter published in Gut by Effenberger et al. ${ }^{42}$.

\section{First IBD diagnosis}

If a new diagnosis of IBD is highly suspected (and supported by cross-sectional imaging for Crohn's disease), colonoscopy or proctosigmoidoscopy with biopsies are

Patient with IBD in COVID-19 pandemic

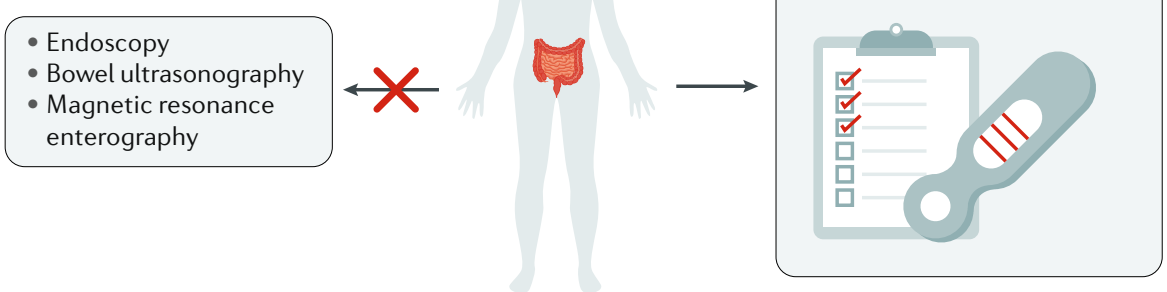

- Patient-reported outcomes - Faecal calprotectin rapid test at home

Fig. 3 | Monitoring IBD disease activity during the COVID-19 pandemic. During the coronavirus disease 2019 (COVID-19) pandemic, home faecal calprotectin tests, where available, together with remote monitoring of symptoms and patient-reported outcomes, could be a safe alternative to monitor patients and could have a key role in avoiding endoscopy or cross-sectional imaging when these procedures are not urgent or necessary. Patient-reported outcomes can be collected with specific patient questionnaires, reporting the number of bowel movements and abdominal pain for Crohn's disease or bowel movements and rectal bleeding for ulcerative colitis. IBD; inflammatory bowel disease. 
indicated to confirm the diagnosis, assess endoscopic activity and extension of disease, and initiate the appropriate therapy. In patients with mild symptoms, the endoscopic procedures should be postponed, as treatment might also be delayed.

In patients with suspected Crohn's disease and isolated small bowel manifestations, without obstructive symptoms or bleeding, enteroscopy should be postponed. If necessary to assess a diagnosis and commence treatment, capsule enteroscopy could be an option $^{27}$, considering that the patient will have to attend the hospital twice with this approach, first for administration of the patency capsule and a second time for capsule endoscopy. In that scenario, all the adequate safety measures will be respected.

\section{Acute severe ulcerative colitis}

Acute severe ulcerative colitis (ASUC) is a medical emergency occurring in about $20-30 \%$ of patients with ulcerative colitis during their lifetime ${ }^{43}$. Multiple definitions of ASUC exist; the first was proposed by Truelove and Witts in 1955 (REF. ${ }^{44}$ ), who suggested a list of criteria to assess patients on initial admission to hospital and to predict the risk of colectomy. The criteria were stool frequency ( $\geq 6$ per day), blood in the stool, heart rate $(>90 \mathrm{bpm})$, temperature $\left(>37.8^{\circ} \mathrm{C}\right)$, haemoglobin levels $(<105 \mathrm{~g} / \mathrm{L})$ and CRP levels $(>30 \mathrm{mg} / \mathrm{L})$. The risk of colectomy was directly correlated with the number of variables present at 1, 3 and 5 days and was $50 \%$ when three or more criteria were present ${ }^{44}$. In 1996, Travis et al. proposed the Oxford score, evaluating only a few parameters to re-assess after 3 days of treatment in patients treated with intravenous corticosteroid as first-line therapy ${ }^{45}$. In this case, to determine the risk of colectomy, the researchers recommended assessment of only stool frequency and serum CRP levels ( $>8$ stools per $24 \mathrm{~h}$ or $3-8$ stools per $24 \mathrm{~h}$ and CRP levels $>45 \mathrm{mg} / \mathrm{L}$ ).

In the COVID-19 era, if a patient with ulcerative colitis is admitted to hospital with bloody diarrhoea, fever and high serum CRP level, it is probably ASUC, yet these symptoms could be similar to the gastrointestinal manifestations of COVID-19; notably, rectal bleeding has been described in 4\% (2 of 52) and $13.7 \%$ (10 of 73) of patients with COVID-19 (REF. ${ }^{18}$ ). In some cases of COVID-19, gastrointestinal symptoms are associated with respiratory symptoms, usually after an initial period of 1-2 days with isolated gastrointestinal symptoms ${ }^{46}$. At the Humanitas clinical centre emergency room, every patient, regardless of respiratory symptoms, has a nasal swab taken and tested to exclude COVID-19 before hospitalization. Differential diagnosis with gastrointestinal infections, such as $C$. difficile infection, is crucial. If the suspicion of ASUC is high, endoscopy is useful to confirm an ulcerative colitis flare, to exclude other diagnoses and to perform concomitant biopsies for cytomegalovirus (CMV) infection. CMV-positive patients with IBD have a higher risk (nearly double) of steroid resistance than CMV-negative patients with IBD as well as a worse prognosis $^{47}$. Several methods for analysing CMV infection in tissue are available; the gold standard for the diagnosis of CMV infection is related to colonic biopsies (haematoxylin and eosin staining via detection of inclusion bodies, so-called owl's eyes, and/or immunohistochemistry assay on histology) rather than serology (antigen or virus detection in blood) ${ }^{48}$. In 2018, PCR-based CMV detection was also applied to evaluate CMV infection in tissue from patients with IBD. This test was highly sensitive (65-100\%) but had limited specificity $(40-92 \%)^{49,50}$.

The SFED advise to keep performing flexible proctosigmoidoscopy in this setting, even in the context of the COVID-19 pandemic ${ }^{33}$. At Humanitas, proctosigmoidoscopies for ASUC are also still performed.

\section{Postoperative recurrence assessment} After surgery, both in Crohn's disease and ulcerative colitis, there is an indication to perform endoscopy to assess endoscopic disease activity. In patients with Crohn's disease after ileocaecal resection, endoscopic recurrence could precede clinical manifestations; indeed, ileocolonoscopy is recommended at 6-12 months from surgery ${ }^{51,52}$.

Endoscopy also plays an important part in the postoperative management of patients with ulcerative colitis. In these patients, proctocolectomy with ileal pouch-anal anastomosis represents the procedure of choice in cases of colitis refractory to treatment or high-grade dysplasia on random biopsy samples according to the European Crohn's and Colitis Organisation guidelines $^{53}$. Endoscopic surveillance of the pouch after surgery is a controversial issue; however, a so-called screening pouchoscopy with biopsies at 1 year from surgery is recommended to assess pouch inflammation and histological activity ${ }^{53}$. After first endoscopy, patients are stratified according to several risk factors such as dysplasia or cancer identified before or at operation time and concomitant diagnosis of primary sclerosing cholangitis (PSC) ${ }^{54}$.

In the COVID-19 era, endoscopy to check postoperative recurrence could be delayed. The possible alternative is to check inflammation activity by performing non-invasive tests, such as a faecal calprotectin home test. In symptomatic patients with Crohn's disease, an accurate differential diagnosis is the first step (gastrointestinal infection, COVID-19, irritable bowel syndrome symptoms) using faecal and haematological tests and PRO measures (by specific questionnaires filled in by the patient). If there is an indication to start therapy, the alternative to endoscopy could be performing bowel ultrasonography to assess disease activity, localization and extension of disease $\mathrm{e}^{55}$. Compared with endoscopy and MRE, bowel ultrasonography needs less time to be performed (40 $\mathrm{min}$ versus 15-20 min; patient spends less time at the hospital), no preparation (bowel preparation, causing diarrhoea and/or vomiting, could increase the risk of spreading COVID-19) and the patient can attend hospital alone (for colonoscopy with sedation it is necessary that a caregiver takes the patient home).

\section{Screening for dysplasia}

For colorectal surveillance and screening of dysplasia, endoscopies planned according to the schedule recommended by the European Crohn's and Colitis Organisation guidelines ${ }^{51}$ and postponed during the lockdown in patients without alarming signs will be rescheduled in the next months. Indeed, in May 2020, lockdown restrictions are starting to be lifted. During the lockdown we could not use laboratory markers for colorectal cancer surveillance because they are not currently available and standard faecal tests (that is, immunological tests such as enzyme-linked immunosorbent assay (ELISA)) for screening of colorectal cancer used for the general population cannot be used in patients with $\mathrm{IBD}^{56}$; indeed, patients with IBD are excluded from national screening programmes. Thus, faecal tests cannot be an alternative for dysplasia screening in patients with IBD during the pandemic and the procedures will have to be rescheduled once it ends.

However, Ten Hove et al. demonstrated, in a large surveillance cohort of 775 patients with IBD with colonic disease and no additional high-risk features, that two consecutive negative colonoscopies predict a very low risk of colorectal cancer occurrence on follow-up ${ }^{57}$. The interval 
between the first colonoscopy and the subsequent surveillance examination was 2.2 years and the duration of follow-up after the first surveillance procedure was 6.1 years. Once the COVID-19 emergency is over and endoscopic departments reopen for patients with IBD, there will be a high number of procedures to recover; thus, a change in surveillance intervals could be useful to better manage the endoscopy department's new agenda in prioritizing patients ${ }^{57}$. Indeed, during the COVID-19 pandemic, a new stratification of patients could be an option in a selected population and might be safe, excluding high-risk patients such as those with PSC or patients with previous dysplastic lesions. If the pandemic lasts more than 3 months we suggest not to further delay surveillance colonoscopy for patients with IBD and concomitant PSC or with prior dysplasia because a time frame longer than 3 months would not be acceptable given the risk of colorectal cancer development. In that case, these patients will be considered as a priority for endoscopic examination.

\section{Endoscopic dilatation}

Obstructive symptoms, such as vomiting, nausea and abdominal pain, require a rapid intervention. In patients with Crohn's disease with a known stricture, management is generally conservative: gastrointestinal nasogastric decompression, bowel rest, intravenous fluids and electrolyte replacement, guided by test results ${ }^{58}$. In cases of repeated obstructive episodes, if the stricture is $<4 \mathrm{~cm}$, endoscopic dilatation is a therapeutic option. If severe and disabling obstructive symptoms are present, a planned endoscopic dilatation should be performed to avoid intestinal occlusion and subsequent admission to the emergency room. In the COVID-19 era, symptomatic patients might have to attend a hospital that is not dedicated to IBD because of movement restrictions, with the risk that the acute obstructive situation could suggest an indication to surgery to a physician not experienced in IBD. Thus, instead of surgery, which requires an anaesthesiologist and surgical teams available and several days for recovery and discharge, endoscopic dilatation is the best management option.

\section{Endoscopic procedures}

The suggestion is to limit upper gastrointestinal procedures, if possible, because of the higher risk of virus transmission in cases of asymptomatic COVID-19-positive patients.

Oesophagogastroduodenoscopies are indicated in cases of acute gastrointestinal bleeding or for dilatation of symptomatic stenosis in the upper gastrointestinal tract in patients with Crohn's disease ${ }^{33}$. For colonoscopy, if the endoscopic procedure is not deferrable, proctosigmoidoscopy instead of full colonoscopy might be preferred, depending on the clinical situation. Proctosigmoidoscopy can reduce the procedure time, the need for sedation and the need for oral bowel preparation.

\section{Endoscopy in industry-sponsored clinical trials}

In many trials, the recruitment of new patients and screening visits have been discontinued by their sponsors. The status of monitoring colonoscopies for participants already recruited in clinical trials should be discussed at the local level and with trial sponsors. If the execution of endoscopy is mandatory to retain patients in the clinical trial, endoscopy might be performed. The investigational product could be the only therapeutic option for the patient and the alternative, like surgery, could be more challenging in the current pandemic. However, the benefits of avoiding surgery and/or corticosteroids by receiving trial medication must be balanced against the risk of the unknown effects of the investigational product on the course of COVID-19. Planned trial visits, whenever possible, should occur virtually and investigation tools could be shipped to patients' homes with specific protocol amendments ${ }^{21,27}$.

\section{Humanitas and SFED recommendations Proposed recommendations and solutions} Based on the Italian and French experience, we propose some recommendations for every indication of endoscopy in patients with IBD (TABLE 1). In brief, we propose the performance of endoscopic examinations only for those patients for whom endoscopy is required for the commencement of appropriate treatment. The justification is that appropriate and timely treatment will decrease the time of hospitalization, or even the need for hospitalization, whilst avoiding disease complications associated with IBD. Proctosigmoidoscopies for ASUC, severe ulcerative colitis flares, and ulcerative colitis and Crohn's disease first diagnosis (excluding mild cases) should be performed, if possible, in the centre. For moderate to severe Crohn's disease flares, alternative faecal calprotectin tests and MRI or ultrasonography should be discussed (TABLE 1). Therapeutic endoscopies for bowel dilatation will also be maintained in symptomatic occlusive patients.

\section{Alternatives to hospital visits Telemonitoring and faecal calprotectin} tests at home. A combination of PROs with faecal calprotectin home test could accurately predict the presence and severity of endoscopic lesions in patients with IBD. However, all non-emergency outpatient visits for patients with IBD should be delayed or cancelled during the COVID-19 pandemic. Furthermore, standard faecal calprotectin tests require a technician to manipulate the stools to perform the test and to collect the stools from the patient; in French university hospitals, it is recommended not to collect stool samples during the pandemic ${ }^{33}$. Telemonitoring, using specific questionnaires and a faecal calprotectin home test, has been studied prior to the COVID-19 era to manage patients with IBD at home and seems safe as conventional follow-up in both paediatric ${ }^{59}$ (teenagers) and adult populations ${ }^{60}$. In a paediatric population study, 170 children and teenagers aged $10-19$ years with IBD in clinical remission at baseline were randomly assigned to follow-up by conventional visit or telemonitoring for 1 year; no statistically significant difference was observed between the two groups in terms of flare or change in quality of life $\mathrm{e}^{59}$. In an adult population study, 180 patients with IBD were randomly assigned into either a home monitoring group (patients performed a faecal calprotectin home test and completed a symptom questionnaire) or a control group (patients had their follow-up visit in the IBD centre); the study duration was 12 months and the disease course between the two groups was similar. In the home monitoring group, patients with a higher disease burden were more adherent than those with a better health-related quality of life ${ }^{60}$. The specific questionnaires used measured PRO, which are important for symptom management. Previously, a PRO measure (Monitor IBD At Home questionnaire or MIAH) was proposed to predict endoscopic inflammation in patients with IBD $^{61}$. The MIAH combined with a faecal calprotectin home test compared with endoscopy showed an excellent diagnostic accuracy (sensitivity of $96.7 \%$ and specificity of $66.7 \%$ for patients with Crohn's disease, and sensitivity of $88.2 \%$ and specificity of $81.4 \%$ for patients with ulcerative colitis).

In summary, telemonitoring seems safe, effective and accurate. In the COVID-19 era, telemonitoring could be an optimal instrument to monitor disease activity, delaying endoscopy when possible. Head-to-head comparison of three different faecal calprotectin home tests, 
Table 1 | Proposed IBD endoscopy recommendations during the COVID-19 outbreak from Humanitas and the SFED

\begin{tabular}{|c|c|c|}
\hline Setting & Proposed recommendation & Proposed solution \\
\hline \multirow[t]{2}{*}{ General recommendation } & \multirow[t]{2}{*}{ Correct PPE for patients and $\mathrm{HCPs}$} & Checkpoints at the hospital or unit entrance \\
\hline & & Correct PPE: mask, gloves, hairnet, gown, hand disinfection \\
\hline $\begin{array}{l}\text { Endoscopy for monitoring } \\
\text { of disease activity }\end{array}$ & Check clinical activity and use non-invasive tests & $\begin{array}{l}\text { Phone call at home by dedicated staff and faecal } \\
\text { calprotectin test at home }\end{array}$ \\
\hline Acute severe ulcerative colitis & Accurate differential diagnosis, biopsies & Maintain proctosigmoidoscopy \\
\hline $\begin{array}{l}\text { Postoperative recurrence } \\
\text { assessment }\end{array}$ & $\begin{array}{l}\text { Reschedule the endoscopic exam for after the end } \\
\text { of pandemic emergency }\end{array}$ & $\begin{array}{l}\text { In symptomatic patients, replace endoscopy with faecal } \\
\text { calprotectin tests and/or bowel ultrasonography }\end{array}$ \\
\hline $\begin{array}{l}\text { Endoscopy for screening } \\
\text { of dysplasia }\end{array}$ & $\begin{array}{l}\text { Reschedule the endoscopic exam for after the end } \\
\text { of pandemic emergency }\end{array}$ & No specific markers are available to avoid endoscopy \\
\hline Endoscopic dilatation & $\begin{array}{l}\text { To be performed if severe and disabling obstructive } \\
\text { symptoms are present }\end{array}$ & $\begin{array}{l}\text { Management of patients in dedicated IBD centres to avoid } \\
\text { surgery }\end{array}$ \\
\hline $\begin{array}{l}\text { Endoscopic procedures for } \\
\text { mild-moderate disease: Crohn's } \\
\text { disease and ulcerative colitis }\end{array}$ & $\begin{array}{l}\text { Limit procedures to decrease the risk of } \\
\text { SARS-CoV-2 transmission to the patient and/or staff }\end{array}$ & Monitor disease with PROs, faecal calprotectin home tests \\
\hline $\begin{array}{l}\text { Endoscopic procedure for } \\
\text { moderate-severe disease: } \\
\text { ulcerative colitis }\end{array}$ & $\begin{array}{l}\text { Maintain endoscopic procedure in situations that } \\
\text { will lead to a therapeutic change: confirmation of } \\
\text { IBD diagnosis, confirmation severe flare }\end{array}$ & Preference for proctosigmoidoscopy to colonoscopy \\
\hline \multirow{2}{*}{$\begin{array}{l}\text { Endoscopic procedure for } \\
\text { moderate-severe disease: } \\
\text { Crohn's disease }\end{array}$} & \multirow{2}{*}{$\begin{array}{l}\text { Maintain endoscopic procedure in situations that } \\
\text { will lead to a therapeutic change: confirmation of } \\
\text { IBD diagnosis, confirmation severe flare }\end{array}$} & $\begin{array}{l}\text { In first Crohn's disease diagnosis: first screen with CT or } \\
\text { MRE, then confirm with colonoscopy and biopsies }\end{array}$ \\
\hline & & $\begin{array}{l}\text { If Crohn's disease is already known: evaluate for faecal } \\
\text { calprotectin tests and/or MRE or bowel ultrasonography }\end{array}$ \\
\hline
\end{tabular}

compared with measurement of calprotectin concentration with the ELISA method by two experienced laboratory technicians, agreed sufficiently ${ }^{62}$. The disadvantages of a faecal calprotectin home test are related to stool manipulation because stool handling is required to add a sample to the test kit. In French university hospitals it is recommended to no longer collect stool samples because of the risk of contagious SARS-CoV-2 transmission. Moreover, telemonitoring could be misleading as an alternative to endoscopy because high faecal calprotectin levels could be unrelated to IBD activity and symptoms; for instance, abdominal pain or diarrhoea could be related to irritable bowel syndrome or other clinical conditions.

Cross-sectional imaging. Bowel

ultrasonography and MRE are non-invasive and well-tolerated techniques shown to have the same level of accuracy in assessing and monitoring Crohn's disease and ulcerative colitis disease activity compared with ileocolonoscopy ${ }^{55}$. CT and CT enterography are also accurate, in particular in assessing Crohn's disease complications ${ }^{51}$, such as fistulae, strictures and abscesses, or in first Crohn's disease diagnosis during the COVID-19 pandemic. CT is fast and readily available, but it exposes individuals to ionizing radiation; therefore, current guidelines ${ }^{51}$ recommend limiting its use. In patients with ulcerative colitis, bowel ultrasonography is useful for monitoring the disease course, for assessing short-term treatment response after 3 or 6 months (is it possible for the exam to be performed by gastroenterologists during the follow-up visit) and it has the potential to predict the therapeutic response ${ }^{63}$. During the COVID-19 pandemic, bowel ultrasonography could become an excellent ally to assess Crohn's disease activity ${ }^{55}$. However, patients still have to attend to hospital to undergo bowel ultrasonography, as with endoscopy, although bowel preparation or sedation are not necessary and fasting $6 \mathrm{~h}$ before the imaging exam is sufficient. Furthermore, bowel ultrasonography has the advantage of a rapid assessment of disease activity (localization, extension, grade of inflammation, presence of complications such as fistula, strictures or intrabdominal abscesses). Unfortunately, bowel ultrasonography is not a widespread technique worldwide; therefore, where available, MRE can give a good assessment of disease activity ${ }^{55}$ and is also non-invasive. In all cases using cross-sectional imaging, the disadvantage is the impossibility of performing biopsies and observing the mucosa directly. These types of only imaging allow assessment of indirect signs of inflammation, such as bowel wall thickening and vascularization, mesenteric inflammation, and the presence of enlarged lymph nodes, or complications related to the disease (obstruction, fistula, abscesses).

As recommended by the European Association of Cardiovascular Imaging, to decrease the risk of peri-procedural transmission of SARS-CoV-2 between patient and health-care staff, the indication for any imaging test should be carefully considered and only tests that are 'essential' to a patient and could change patient management or care should be performed $^{64}$. Ultrasonography, MRE and CT have the potential for contamination of hospital personnel and patients by direct contact, by the contamination of equipment and facilities (all equipment has the potential to carry droplets), by patient transportation or when the procedure is time consuming (higher for MRE). To clean the instrument, including the probes, warm water, a mild detergent and water-soluble disinfectant are suggested; alternatively, dedicated disinfectant wipes could be an option. The suggested PPE is the same as used in endoscopy both for HCPs and for patients. To reduce contamination, for known COVID-19-positive patients, a good strategy is to reserve a dedicated MRE, CT or ultrasonography scanner ${ }^{64}$. 


\section{Conclusions}

The protection of patients with IBD requiring endoscopy is a key point to consider in their daily IBD management. Measures of prevention should be planned and adopted in all endoscopy units that manage patients with and without IBD. In the management of IBD-specific endoscopic situations, alternative biomarkers replacing non-urgent endoscopy, such as faecal calprotectin home test to assess disease activity, should be considered and implemented. To understand whether endoscopy could be rescheduled, it is also very important to maintain close contact with patients by phone or e-mail to monitor for specific symptoms (through the use of standardized questionnaires) and general clinical condition as well as for indications of SARS-CoV-2 infection.

Federica Furfaro ${ }^{1}$, Lucine Vuitton ${ }^{2}$, Gionata Fiorino ${ }^{1,3}$, Stephane Koch2 ${ }^{2}$, Mariangela Allocca ${ }^{1,3}$,

Daniela Gilardi ${ }^{1}$, Alessandra Zilli ${ }^{1}$

Ferdinando D'Amico ${ }^{3,4}$, Simona Radice',

Jean-Baptiste Chevaux 4 , Marion Schaefer

Stanislas Chaussade ${ }^{5}$, Silvio Danese (D) ${ }^{1,3}$ and Laurent Peyrin-Biroulet iD 4 凶

'IBD Center, Department of Gastroenterology, Humanitas Clinical and Research Center - IRCCS, Rozzano, Italy.

${ }^{2}$ Department of Gastroenterology, Besançon University Hospital, Bourgogne-Franche-Comté University, Besançon, France.

${ }^{3}$ Department of Biomedical Sciences, Humanitas University, Pieve Emanuele, Italy.

${ }^{4}$ Department of Gastroenterology and Inserm NGERE U1256, University Hospital of Nancy, University of Lorraine, Vandoeuvre-lès-Nancy, France.

${ }^{5}$ Department of Gastroenterology, Paris Cochin University Hospital APHP, Paris 5 University, Besançon, France.

凶e-mail:peyrinbiroulet@gmail.com

https://doi.org/10.1038/s41575-020-0319-3

Published online 11 June 2020

1. Fiorino, G. et al. Quality of care standards in inflammatory bowel diseases: a European Crohn's and Colitis Organisation (ECCO) position paper. J. Crohns Colitis https://doi.org/10.1093/ecco-jcc/jjaa023 (2020).

2. Peyrin-Biroulet, L. et al. Selecting therapeutic targets in inflammatory bowel disease (STRIDE): determining therapeutic goals for treat-to-target. Am. J. Gastroenterol. 110, 1324-1338 (2015).

3. Gorbalenya, A. E. et al. The species severe acute respiratory syndrome-related coronavirus: classifying 2019-nCoV and naming it SARS-CoV-2 Nat. Microbiol. 5, 536-544 (2020).

4. Zhu, N. et al. A novel coronavirus from patients with pneumonia in China, 2019. N. Engl. J. Med. 382 727-733 (2020)

5. Fiorino, G. et al. Inflammatory bowel disease care in the COVID-19 pandemic era: the Humanitas, Milan experience. J. Crohns Colitis https://doi.org/10.1093/ ecco-jcc/jjaa058 (2020).

6. World Health Organization. COVID-19 Situation in the WHO European Region https://who.maps. arcgis.com/apps/opsdashboard/index.html\#l ead3c6475654481ca51c248d52ab9c61 (2020)

7. Cui, J., Li, F. \& Shi, Z. L. Origin and evolution of pathogenic coronaviruses. Nat. Rev. Microbiol. 17, 181-192 (2019)

8. Meo, S. A. et al. Novel coronavirus 2019-nCoV: prevalence, biological and clinical characteristics comparison with SARS-CoV and MERS-CoV. Eur. Rev. Med. Pharmacol. Sci. 24, 2012-2019 (2020).

9. Radonovich, L. J. Jr. et al. N95 respirators vs medical masks for preventing influenza among health care personnel: a randomized clinical trial. JAMA 322 824-833 (2019)

10. Gao, Q. Y., Chen, Y. X. \& Fang, J. Y. 2019 novel coronavirus infection and gastrointestinal tract. J. Dig. Dis. 21, 125-126 (2020).

11. D'Amico, F., Baumgart, D. C., Danese, S. \& Peyrin-Biroulet, L. Diarrhea during COVID-19 infection: pathogenesis, epidemiology, prevention and management. Clin. Gastroenterol. Hepatol. https://doi.org/10.1016/j.cgh.2020.04.001 (2020).

12. Wölfel, R. et al. Virological assessment of hospitalized patients with COVID-2019. Nature https://doi.org/ 10.1038/s41586-020-2196-x (2020).

13. Wang, W. et al. Detection of SARS-CoV-2 in different types of clinical specimens. JAMA https://doi.org/ 10.1001/jama.2020.3786 (2020).

14. Xiao, F. et al. Evidence for gastrointestinal infection of SARS-CoV-2. Gastroenterology https://doi.org/ 10.1053/j.gastro.2020.02.055 (2020)

15. Gu, J., Han, B. \& Wang, J. COVID-19: gastrointestinal manifestations and potential fecal-oral transmission. Gastroenterology https://doi.org/10.1053/j.gastro. 2020.02.054 (2020).

16. Ong, S. W. X. et al. Air, surface environmental, and personal protective equipment contamination by severe acute respiratory syndrome coronavirus 2 (SARS-CoV-2) from a symptomatic patient. JAMA https://doi. org/10.1001/jama.2020.3227 (2020).

17. Lamers, M. M. et al. SARS-CoV-2 productively infects human gut enterocytes. Science https:// doi.org/10.1126/science.abc1669science.abc 1669 (2020).

18. Tian, Y., Rong, L., Nian, W. \& He, Y. Review article: gastrointestinal features in COVID-19 and the possibility of faecal transmission. Aliment. Pharmacol. Ther. 51, 843-851 (2020).

19. Sultan, S. et al. AGA institute rapid recommendations for gastrointestinal procedures during the COVID-19 pandemic. Gastroenterology https://doi.org/10.1053/ j.gastro.2020.03.072 (2020).

20. Wang, P. et al. Rates of infection after colonoscopy and osophagogastroduodenoscopy in ambulatory surgery centres in the USA. Gut 67, 1626-1636 (2018).

21. Kennedy, N. et al. British Society of Gastroenterology guidance for management of inflammatory bowel disease during the COVID-19 pandemic. Gut https:// doi.org/10.1136/gutjnl-2020-321244 (2020).

22. International Organization for the Study of Inflammatory Bowel Disease (IOIBD). IOIBD Update on COVID 19 for Patients with Crohn's Disease and Ulcerative Colitis https://www.ioibd.org/ioibd-updateon-covid 19-for-patients-with-crohns-disease-andulcerative-colitis/ (2020).

23. European Society of Gastrointestinal Endoscopy $\&$ European Society of Gastroenterology and Endoscopy Nurses and Associates. ESGE and ESGENA Position Statement on Gastrointestinal Endoscopy and the COVID-19 Pandemic https://www.esge.com/esgeand-esgena-position-statement-on-gastrointestinalendoscopy-and-the-covid-19-pandemic/ (2020).

24. Mao, R. et al. Implications of COVID-19 for patients with pre-existing digestive diseases. Lancet Gastroenterol. Hepatol. 5, 426-428 (2020).

25. Chiu, P. W. Y. et al. Practice of endoscopy during COVID-19 pandemic: position statements of the Asian Pacific Society for Digestive Endoscopy (APSDE-COVID statements). Gut https://doi.org 10.1136/gutjnl-2020-321185 (2020)

26. Perisetti, A., Gajendran, M., Boregowda, U. Bansal, P. \& Goyal, H. COVID-19 and gastrointestinal endoscopies: current insights and emergent strategies. Dig. Endosc. https://doi.org/10.1111/den.13693 (2020).

27. Iacucci, M. et al. Endoscopy in inflammatory bowel diseases during the COVID-19 pandemic and postpandemic period. Lancet Gastroenterol. Hepatol. https://doi.org/10.1016/s2468-1253(20)30119-9 (2020).

28. John Hopkins University Coronavirus Resource Center. Coronavirus COVID-19 Global Cases https://coronavirus.jhu.edu/map.html (2020)

29. World Health Organization. Report of the WHO-China Joint Mission on Coronavirus Disease 2019 (COVID-19) https://www.who.int/docs/default-source/ coronaviruse/who-china-joint-mission-on-covid-19 final-report.pdf (2020).

30. Pan, L. et al. Clinical characteristics of COVID-19 patients with digestive symptoms in Hubei, China: a descriptive, cross-sectional, multicenter study. Am. J. Gastroenterol 115, 766-773 (2020).

31. Berenger, B. M., Fonseca, K., Schneider, A. R., Hu, J. $\ltimes$ Zelyas, N. Sensitivity of nasopharyngeal, nasal and throat swab for the detection of SARS-CoV-2. Preprint at MedRxiv https://doi.org/10.1101/ 2020.05.05.20084889 (2020).

32. Leung, N. H. L. et al. Respiratory virus shedding in exhaled breath and efficacy of face masks. Nat. Med. https://doi.org/10.1038/s41591-020-0843-2 (2020).

33. Société Française d'Endoscopie Digestive. COVID-19 et endoscopie: informations et alertes pour les professionnels. Société Française d'Endoscopie Digestive https://www.sfed.org/professionnels/ covid19-et-endoscopie (2020).

34. van Doremalen, N. et al. Aerosol and surface stability of SARS-CoV-2 as compared with SARS-CoV-1. N. Engl. J. Med. https://doi.org/10.1056/NEJMc2004973 (2020).

35. European Centre for Disease Prevention and Control. Disinfection of Environments in Healthcare and Nonhealthcare Settings Potentially Contaminated with SARS-CoV-2 https://www.ecdc.europa.eu/sites/default files/documents/Environmental-persistence-of-SARS CoV_2-virus-Options-for-cleaning2020-03-26_0.pdf. (2020).

36. Kampf, G., Todt, D., Pfaender, S. \& Steinmann, E. Persistence of coronaviruses on inanimate surfaces and their inactivation with biocidal agents. J. Hospital Infect. 104, 246-251 (2020).

37. Huang, C. et al. Clinical features of patients infected with 2019 novel coronavirus in Wuhan, China. Lancet 395, 497-506 (2020)

38. Colombel, J.-F. et al. Effect of tight control management on Crohn's disease (CALM): a multicentre, randomised, controlled phase 3 trial. Lancet 390, 2779-2789 (2017).

39. Patel, A., Panchal, H. \& Dubinsky, M. C. Fecal calprotectin levels predict histological healing in ulcerative colitis. Inflamm. Bowel Dis. 23, 1600-1604 (2017).

40. D'Amico, F. Bonovas, S., Danese, S. \& Peyrin-Biroulet, L. Review article: faecal calprotectin and histologic remission in ulcerative colitis. Aliment. Pharmacol. Ther. 51, 689-698 (2020).

41. Jin, X. et al. Epidemiological, clinical and virological characteristics of 74 cases of coronavirus-infected disease 2019 (COVID-19) with gastrointestinal symptoms. Gut https://doi.org/10.1136/ gutjnl-2020-320926 (2020)

42. Effenberger, M. et al. Faecal calprotectin indicates intestinal inflammation in COVID-19. Gut https:/ doi.org/10.1136/gutjnl-2020-321388 (2020).

43. Verdon, C., Bessissow, T. \& Lakatos, P. L. Management of acute severe colitis in the era of biologicals and small molecules. J. Clin. Med. https://doi.org/10.3390/ jcm8122169 (2019).

44. Truelove, S. C. \& Witts, L. J. Cortisone in ulcerative colitis; final report on a therapeutic trial. Br. Med. J. $\mathbf{2}$, 1041-1048 (1955).

45. Travis, S. P. et al. Predicting outcome in severe ulcerative colitis. Gut 38, 905-910 (1996).

46. Wang, D. et al. Clinical characteristics of 138 hospitalized patients with 2019 novel coronavirusinfected pneumonia in Wuhan, China. JAMA 323 1061-1069 (2020).

47. Wu, X.-W., Wu, L., Ji, H.-Z. \& Wang, F-Y. Relationship between cytomegalovirus infection and steroid resistance in inflammatory bowel disease: a meta-analysis. Digestive Dis. Sci. 60, 3203-3208 (2015).

48. Mourad, F. H., Hashash, J. G., Kariyawasam, V. C. \& Leong, R. W. Ulcerative colitis and cytomegalovirus infection: from A to Z. J. Crohns Colitis https://doi.org 10.1093/ecco-jcc/jjaa036 (2020)

49. Wethkamp, N., Nordlohne, E.-M., Meister, V. Helwig, U. \& Respondek, M. Identification of clinically relevant cytomegalovirus infections in patients with inflammatory bowel disease. Mod. Pathol. 31, 527-538 (2018)

50. Lawlor, G. $\&$ Moss, A. C. Cytomegalovirus in inflammatory bowel disease: pathogen or innocent bystander? Inflamm . Bowel Dis. 16, 1620-1627 (2010).

51. Maaser, C. et al. ECCO-ESCAR guideline for diagnostic assessment in IBD part 1: initial diagnosis, monitoring of known IBD, detection of complications. J. Crohns Colitis 13, 144-164 (2019).

52. Nguyen, G. C. et al. American gastroenterological association institute guideline on the management of Crohn's disease after surgical resection. Gastroenterology 152, 271-275 (2017). 


\section{PERSPECTIVES}

53. Magro, F. et al. Third European evidence-based consensus on diagnosis and management of ulcerative colitis. part 1: definitions, diagnosis, extra-intestinal manifestations, pregnancy, cancer surveillance, surgery, and ileo-anal pouch disorders. J. Crohns Colitis 11, 649-670 (2017).

54. Annese, V. et al. European evidence-based consensus: inflammatory bowel disease and malignancies. J. Crohns Colitis 9, 945-965 (2015).

55. Allocca, M., Danese, S., Laurent, V. \& Peyrin-Biroulet, L. Use of cross-sectional imaging for tight monitoring of inflammatory bowel diseases. Clin. Gastroenterol. Hepatol. 18, 1309-1323.e4 (2020).

56. Toritani, K. et al. Uselessness of serum p53 antibody for detecting colitis-associated cancer in the era of immunosuppressive therapy. In Vivo 34, 723-728 (2020).

57. Ten Hove, J. R. et al. Consecutive negative findings on colonoscopy during surveillance predict a low risk of advanced neoplasia in patients with inflammatory bowel disease with long-standing colitis: results of a 15-year multicentre, multinational cohort study. Gut 68, 615-622 (2019)

58. Rieder, F. et al. European Crohn's and Colitis Organisation topical review on prediction, diagnosis and management of fibrostenosing Crohn's disease. J. Crohns Colitis 10, 873-885 (2016).

59. Heida, A. et al. Efficacy of home telemonitoring versus conventional follow-up: a randomized controlled trial among teenagers with inflammatory bowel disease. J. Crohns Colitis 12, 432-441 (2018).
60. Puolanne, A. M., Kolho, K. L., Alfthan, H. \& Farkkila, M Is home monitoring of inflammatory bowel disease feasible? A randomized controlled study. Scand. J. Gastroenterol. 54, 849-854 (2019).

61. de Jong, M. J. et al. Development and validation of a patient-reported score to screen for mucosal inflammation in inflammatory bowel disease. J. Crohns Colitis 13, 555-563 (2019)

62. Haisma, S. M. et al. Head-to-head comparison of three stool calprotectin tests for home use. PLoS One 14 e0214751 (2019).

63. Maaser, C. et al. Intestinal ultrasound for monitoring therapeutic response in patients with ulcerative colitis: results from the TRUST\&UC study. Gut https://doi.org/ 10.1136/gutjnl-2019-319451 (2019).

64. Skulstad, H. et al. COVID-19 pandemic and cardiac imaging: EACVI recommendations on precautions, indications, prioritization, and protection for patients and healthcare personnel. Eur. Heart J. Cardiovasc. Imaging 21, 592-598 (2020).

\section{Author contributions}

The authors contributed equally to all aspects of the article.

\section{Competing interests}

The authors disclose the following: F.F. received consultancy fees from MSD, AbbVie, Janssen and Amgen. L.V. has received lecture fees from Abbvie, Ferring, MSD, Pfizer, Janssen and Takeda; consulting fees from Abbvie, Ferring and Gilead; and research grants from MSD, Pfizer and Takeda. G.F. received consultancy fees from Ferring, MSD, AbbVie, Takeda, Janssen, Amgen, Sandoz, Samsung Bioepis,
Celltrion, Mylan and Roche. S.K. has received lecture fees from Abbvie, Mayoli, MSD, FujiFilm. M.A. received consultancy fees from Nikkiso Europe and lecture fees from Janssen, Abbvie and Pfizer. D.G. received consultancy fees from Nikkiso, Sofar Spa, Boiler Spa, JळJ, Pfizer, Takeda and Roche. S.D. has served as a speaker, consultant and advisory board member for Schering-Plough, Abbott (AbbVie) Laboratories, Merck, UCB Pharma, Ferring, Cellerix, Millenium Takeda, Nycomed, Pharmacosmos, Actelion, a Wasserman, Genentech, Grunenthal, Pfizer, AstraZeneca, Novo Nordisk, Cosmo Pharmaceuticals, Vifor and Johnson \& Johnson. L.P.B. has received consulting fees from Merck, AbbVie, Janssen, Genentech, Mitsubishi, Ferring, Norgine, Tillots, Vifor, Therakos, Pharmacosmos, Pilège, BMS, UCB pharma, Hospira, Celltrion, Takeda, Biogaran, Boerhinger Ingelheim, Lilly, Pfizer, HAC-Pharma, Index Pharmaceuticals, Amgen, and Sandoz and lecture fees from Merck, AbbVie, Takeda, Janssen, Takeda, Ferring, Norgine, Tillots, Vifor, Therakos, Mitsubishi and HAC pharma. A.Z., F.D., J.-B.C., M.S., S.C. and S.R. declare no competing interests.

Peer review information

Nature Reviews Gastroenterology \& Hepatology thanks R. Atreya, A. Dignass and J. D. Feuerstein for their contribution to the peer review of this work.

\section{Publisher's note}

Springer Nature remains neutral with regard to jurisdictional claims in published maps and institutional affiliations.

(C) Springer Nature Limited 2020 